\title{
Josephson detector of gravitational waves: non-formal consequence of formal analogy: critical wave length - critical current
}

\author{
Zherikhina L.N., Petrova M.G., Tskhovrebov A.M. \\ P.N.Lebedev Physical Institute of the RAS, Moscow, Russia; \\ E-mail: Zherikhina<zherikh@sci.lebedev.ru>;
}

The DC-SQUID as the gravitational wave detector is offered. It is proposed to use the Josephson tunneling, and register a broadening of the gap between the superconducting needle and the plate rigidly connected with a transducer. For supersensitivity the suggested increasing of steepness for the dependence between Josephson inductance - current when the external current approaches the critical value is noted. Some estimates of its sensitivity on amplitude for 1 $\mathrm{GHz}$ range wave frequency is presented.

Keywords: gravitational waves, SQUID, interferometric detectors.

DOI: $10.18698 / 2309-7604-2015-1-577-580$

In [1] it was proposed to explore high despersion of light propagating in narrow channel for registration of gravitational waves. When wavelength of light $\lambda_{0}$ is close to the critical wavelength for a given channel $\lambda_{C}$ its value strongly depends upon the transverse dimension of the channel $w$. When the last is changed by a gravitational disturbance the wavelength of light inside the channel occurs sensitive to these variations. The necessary sensitivity of gravitational waves detector is evaluated on the level of $\left|\delta g_{i j}^{\perp}\right| \approx \delta h / h=\delta w / h \approx\left(10^{-20} \div 10^{-22}\right) / \sqrt{H z}$. Due to high dispersion of light with wavelength $\lambda_{0} \approx \lambda_{C}$ even very small relative changing of channel width $w$ could cause alteration of optical way for electromagnetic wave of definite type that could be noticed with the help of interferometric methods. Relative elongation of massive cylinder antenna $\delta h / h$ is approximately equal to he amplitude of transverse gravitational wave. Hence if one arrange a channel one side of will be the mirror attached to the bull-end of the gravitational wave detector antenna and direct light into this waveguide its wavelength should enhance according with the law $\lambda=\frac{\lambda_{0}}{\sqrt{1-\left(\lambda_{0} / \lambda_{C}\right)^{2}}}$ For the lowest nontransverse type of wave $\lambda_{C}=2 w$. The addition of phase $\delta \varphi$ which is registered interferometricaly is caused by two reasons: direct physics elongation of optic way $\delta \ell_{\text {opt }}$ and indirect - through the change of channel width $w$ in view of $\delta \lambda_{C}=2 \delta w$. Under condition $\ell_{o p t}=$ const $=\ell_{0} \delta \phi=\delta\left(\frac{2 \pi \ell_{0}}{\lambda}\right)=\frac{d}{d \lambda_{C}}\left(\frac{2 \pi \ell_{0}}{\lambda}\right) \delta \lambda_{C}$. Let's rewrite the last equation, having 
expressed the change of critical wavelength through the lengthening of working body of antenna. Then

$$
\begin{aligned}
& \delta \phi=\frac{2 \pi \ell_{0}}{\lambda_{0}} \frac{d}{d \lambda_{C}}\left(\sqrt{1-\left(\lambda_{0} / \lambda_{C}\right)^{2}}\right) \delta \lambda_{C}=\frac{2 \pi \ell_{0}}{\lambda_{0}} \frac{\lambda_{0}^{2} / \lambda_{C}^{3}}{\sqrt{1-\left(\lambda_{0} / \lambda_{C}\right)^{2}}} 2 h\left|\delta g_{i j}^{\perp}\right| \approx \\
& \approx \frac{2 \pi \ell_{0}}{\lambda_{0}} \sqrt{\frac{2 \lambda_{0}}{\lambda_{C}-\lambda_{0}}} \frac{h}{\lambda_{0}}\left|\delta g_{i j}^{\perp}\right|
\end{aligned}
$$

When meter-long lengths of channel $\ell_{0} \approx 1 \mathrm{~m}$ and antenna cylinder of $h \approx 1 \mathrm{~m}$, on operating wavelengths $\lambda_{0} \approx 0,5 \mathrm{mkm}$ with tuning out from critical $\frac{\lambda_{C}-\lambda_{0}}{\lambda_{0}} \approx 0,02 \%$ the gravitational wave by the amplitude $\left|\delta g_{i j}^{\perp}\right| \approx 4 \times 10^{-21} / \sqrt{H z}$ is to call in such a system a phase response, which corresponds to sensibility of good Mach -Zander interferometer with megapicsel digital camera. (The tuning out on the level of $0.02 \%$ is quite real. Thus in [2] authors happened to brake electromagnetic wave down to velocity the $15 \mathrm{~m} / \mathrm{sec}$, it means that factor $\sqrt{\frac{2\left(\lambda_{C}-\lambda_{0}\right)}{\lambda_{0}}}$ achieved the value $2 \times 10^{7}$ ). As a result it turns out that the main "sensibility reserve" is determined by the factor $\sqrt{\frac{2 \lambda_{0}}{\lambda_{C}-\lambda_{0}}}$, diverging when zero detuning. The origin of this fact is linked to the law of wavelength conversion in the channel $\lambda=\frac{\lambda_{0}}{\sqrt{1-\left(\lambda_{0} / \lambda_{C}\right)^{2}}}$. A similar expression one can meet in physics of Josephson effect when description of its reactive parameters answering to kinetic energy of dissipationless movement of Cooper pairs while tunneling through potential barrier. Let's put the well known dependence of Josephson current from phase difference on tunneling barrier $I_{J}=I_{J C} \sin \varphi[3-5]$ into Faraday induction law $-U=L \frac{d I}{d t}$ (here $I_{J C}-$ the critical current of tunneling barrier, playing a part of amplitude of Josephson current). Then, meaning that the speed of phase "turning" in Josephson effect is proportional to voltage drop on the barrier, will get the expression for kinetic inductance (i. e. not related to magnetic energy) as follows: 


$$
\begin{aligned}
& L_{J}=\frac{U}{\frac{d}{d t}\left(I_{J C} \sin \phi\right)}=\frac{U}{I_{J C} \cos \phi \frac{d \phi}{d t}}=\frac{U}{I_{J C} \frac{2 e}{\hbar} U \cos \phi}=\frac{\hbar}{2 e I_{J C} \cos \phi}=\frac{(\pi \hbar / e)}{2 \pi I_{J C} \cos \phi}= \\
& =\frac{\Phi_{0}}{2 \pi I_{J C} \cos \phi}
\end{aligned}
$$

where $\Phi_{0}=\pi \hbar / e \approx 2,07 \times 10^{-} 15 \mathrm{~Wb}-$ magnetic flux quantum [6]. Further substitute $I_{J}=I_{J C} \sin \varphi$ into obtained formula and transform it to form $L_{J}(\varphi)=\frac{\Phi_{0}}{2 \pi I_{J C} \cos \varphi}=\frac{\Phi_{0}}{2 \pi I_{J C} \sqrt{1-\sin ^{2} \varphi}}=\frac{\Phi_{0}}{2 \pi I_{J C} \sqrt{1-\left(I_{J} / I_{J C}\right)^{2}}}$, that on structure really resembles $\lambda=\frac{\lambda_{0}}{\sqrt{1-\left(\lambda_{0} / \lambda_{C}\right)^{2}}}$ to a certain extent. Let's express the increment of Josephson inductance $\delta L_{J}$ through the variation of Josephson critical current $\delta L_{J C}$ :

$$
\begin{gathered}
\delta L_{J}=\left.\frac{\partial L_{J}}{\partial I_{J C}}\right|_{I_{J}=\text { const }} \delta I_{J C}=-\frac{\Phi_{0}}{2 \pi I_{J C}^{2}\left(\sqrt{1-\left(\frac{I_{J}}{I_{J C}}\right)^{2}}\right)^{3}} \delta I_{J C}= \\
=-\frac{L}{\left(1-\left(\frac{I_{J}}{I_{J C}}\right)^{2}\right)} \frac{\delta I_{J C}}{I_{J C}} \approx-\frac{L}{2\left(\frac{I_{J C}-I_{J}}{I_{J C}}\right)} \frac{\delta I_{J C}}{I_{J C}}
\end{gathered}
$$

or for relative increments $\frac{\delta L_{J}}{L} \approx-2\left(\frac{I_{J C}-I_{J}}{I_{J C}}\right)^{-1} \frac{\delta I_{J C}}{I_{J C}}$. So that, under relative tuning out from critical current of order of $0,1 \%$ the relationship for relative increments will be $\frac{\delta L_{J}}{L} \approx-2 \times 10^{3} \frac{\delta I_{J C}}{I_{J C}}$. Further, taking into account that $\frac{\delta f}{f} \approx-0,5 \frac{\delta L_{J}}{L}$ (here $f=\frac{1}{2 \pi \sqrt{L_{J} C}}$ frequency of electronic generator with $L_{J} C$ oscillatory circuit, $C=$ const ) and $\frac{\delta I_{J C}}{I_{J C}} \approx-\frac{\delta x}{\xi}$ (where 
$I_{J C} \sim e^{-\frac{x}{\xi}}$ and $\xi$ - typical size of Cooper pair) we get $\frac{\delta f}{f} \approx-10^{3} \frac{\delta x}{\xi}$. Let's consider the Josephson junction between two superconductors with $\xi=1 \mathrm{~nm}$ and organized as point contact of the needle terminated at plate, attached to the butt-end of cylindrical gravitational antenna by the height of $h \approx 1 \mathrm{~m}$. Then change of tunneling barrier width $\delta x$ and elongation of antenna working body $\delta h$ are expressed through the amplitude of gravitational wave as $\delta x=|\delta h|=h\left|\delta g_{i j}^{\perp}\right|$, wherefrom $\frac{\delta f}{f} \approx-10^{3} \frac{h\left|\delta g_{i j}^{\perp}\right|}{\xi} \approx-10^{12}\left|\delta g_{i j}^{\perp}\right|$. It means that when "undisturbed" generation frequency is at the level of $f \approx 10 \mathrm{GHz}$ the fixable variation of frequency would correspond to action of gravitational wave with amplitude $\left|\delta g_{i j}^{\perp}\right| \approx 10^{-22}$. It should be noticed that owing to quasi nondissipativity of processes in Josephson junction when $I<I_{J C}$ (in zero approach voltage drop on the junction is equal to zero) temperature of Nyquist noise, determining the intensity of phase-frequency fluctuations of generator (where frequency setting $L_{J} C$ circuit is included) will occur at the level essentially lower, than the physics temperature of tunnel junction. It is clear that the data the circumstance is additional and highly essential factor facilitating achievement of super high sensitivity of system necessary for gravitational waves detector. Nondissipativity and increasing of parametric response, corresponding to little tuning out of operating current from critical one, make this system essentially preferable, in comparison with numerous proposals to create the gravitational wave detector based on tunneling microscope.

The work was supported in frameworks of the program "Strongly correlated electrons in semiconductors, metals, superconductors and magnetic materials” of RAS (the project № II-3 ).

\section{References}

1. Zherikhina L.N., Petrova M.G., Tskhovrebov A.M., Berlov I.V. (2013). Bulletin of the Lebedev Physics Institute, 40, №6, 31-41.

2. Gorelik V.S. (2008). Laser Physics, 3(12), 1479.

3. Josephson B.D. (1962). Phys. Lett., 1, 251.

4. Josephson B.D. (1974). Rev. Mod. Phys., 46, 251.

5. Anderson P.W. (1970). Phys. Today, 23, 20.

6. Golovashkin A.I., Elenskii V.G., Likharev K.K. (1983). Josephson effect and its application. Moscow: Nauka [Science]. 